\title{
A Nonlinear Programming Approach to Optimal Design Centering, Tolerancing, and Tuning
}

\author{
JOHN W. BANDLER, SENIOR MEMBER, IEEE, PETER C. LIU, MEMBER, IEEE, AND HERMAN TROMP
}

\begin{abstract}
A theory of optimal worst-case design embodying centering, tolerancing, and tuning is presented. Some simplified problems and special cases are discussed. Projections and slack variables are used to explain some of the concepts. The worst-case tolerance assignment and design centering problem falls out as a special case. Practical implementation requires a reasonable and relevant number of parameters and constraints to be identified to make the problem tractable. Two circuits, a simple $L C$ low-pass filter and a realistic high-pass filter, are studied under a variety of different problem situations to illustrate both the benefits to be derived from our approach and the difficulties encountered in its implementation.
\end{abstract}

\section{INTRODUCTION}

$\mathrm{C}$ OMPONENT TOLERANCE ASSIGNMENT is now considered to be an integral part of the design process [1]-[7]. The optimal worst-case tolerance problem with variable nominal point has benefitted in terms of increased tolerances [5]-[7]. Tuning [7], [8], on the other hand, does not seem to have been given its proper place in the design process. This work, therefore, brings in tuning of one or more components basically to further increase tolerances to reduce cost or to make unrealistically toleranced solutions more attractive. The mathematical formulation of an approach which embodies centering, tolerancing, and tuning in a unified manner is presented. Simplified problems and appropriate geometric interpretations are discussed. The worst-case purely toleranced problem and purely tuned problem fall out as special cases, as is to be expected. Numerical examples involving simple functions and a realistic as well as a simple circuit, illustrate the concepts.

\section{Fundamental Concepts and Definitions}

A design consists of design data of the nominal point $\phi^{0}$, the tolerance vector $\varepsilon$ and the tuning vector $t$ where, for $k$

Manuscript received November 15, 1974; revised October 30, 1975. This work was supported in part by the National Research Council of Canada under Grant A7239 and in part by a Graduate Fellowship of the Rotary Foundation to H. Tromp. This paper is based on material presented at the 12th Annual Allerton Conference on Circuit and System Theory, Urbana, IL, October 2-4, 1974, and at the 1975 IEEE International Symposium on Circuits and Systems, Newton, Mass., April 21-23, 1975.

J. W. Bandler is with the Group on Simulation, Optimization and Control and the Department of Electrical Engineering, McMaster University, Hamilton, Canada, L8S 4L7.

P. C. Liu was with the. Group on Simulation, Optimization and Control and the Department of Electrical Engineering, McMaster University, Hamilton, Canada. He is now with Bell-Northern Research, Verdun, P.Q., Canada.

H. Tromp was with the Group on Simulation, Optimization and Control and the Department of Electrical Engineering, McMaster University, Hamilton, Canada. He is now with the Laboratory of Electromagnetism and Acoustics University of Ghent, Ghent, Belgium. parameters,

$$
\phi^{0} \triangleq\left[\begin{array}{c}
\phi_{1}{ }^{0} \\
\phi_{2}{ }^{0} \\
\vdots \\
\phi_{k}{ }^{0}
\end{array}\right], \varepsilon \triangleq\left[\begin{array}{c}
\varepsilon_{1} \\
\varepsilon_{2} \\
\vdots \\
\varepsilon_{k}
\end{array}\right] \text {, and } t \triangleq\left[\begin{array}{c}
t_{1} \\
t_{2} \\
\vdots \\
t_{k}
\end{array}\right] .
$$

We assume that the parameters can be varied continuously and chosen independently. Extra conditions such as discretization and imposed parameter bounds may be treated as constraints [6]. Some of the parameters can be set to zero or held constant.

An outcome $\left\{\phi^{0}, \boldsymbol{\varepsilon}, \boldsymbol{\mu}\right\}$ of a design $\left\{\phi^{0}{ }^{0} \boldsymbol{\varepsilon}, \boldsymbol{t}\right\}$ implies a point

$$
\boldsymbol{\phi}=\boldsymbol{\phi}^{0}+\boldsymbol{E} \mu
$$

where

$$
E \triangleq\left[\begin{array}{llll}
\varepsilon_{1} & & & \\
& \varepsilon_{2} & & \\
& & \ddots & \\
& & & \varepsilon_{k}
\end{array}\right]
$$

and $\mu \in R_{\mu} . R_{\mu}$ is a set of multipliers determined from realistic situations of the tolerance spread. For example,

$$
R_{\mu} \triangleq\left\{\boldsymbol{\mu} \mid-1 \leq \mu_{i} \leq-a_{i} \text { or } a_{i} \leq \mu_{i} \leq 1, i \in I_{\phi}\right\}
$$

where

and

$$
0 \leq a_{i} \leq \mathrm{I}
$$

$$
I_{\phi} \triangleq\{1,2, \cdots, k\} \text {. }
$$

The most commonly used continuous range is obtained by setting $a_{i}$ to zero. A commercial stock may have the better toleranced components taken out, thus $0<a_{i} \leq 1$. Unless otherwise stated, we consider

$$
R_{\mu} \triangleq\left\{\mu \mid-1 \leq \mu_{i} \leq 1, i \in I_{\phi}\right\}
$$

The tolerance region $R_{\varepsilon}$ is a set of points described by (2) for all $\mu \in R_{\mu}$. In the case of $-1 \leq \mu_{i} \leq 1, i \in I_{\phi}$,

$$
R_{\varepsilon} \triangleq\left\{\phi \mid \phi_{i}=\phi_{i}{ }^{0}+\varepsilon_{i} \mu_{i},-1 \leq \mu_{i} \leq 1, i \in I_{\phi}\right\}
$$

which is a convex regular polytope of $k$ dimensions with sides of length $2 \varepsilon_{i}, i \in I_{\phi}$, and centered at $\phi^{0}$. The extreme points of $R_{\varepsilon}$ are obtained by setting $\mu_{i}= \pm 1$. Thus, the set of vertices may be defined as

$$
R_{v} \triangleq\left\{\phi \mid \phi_{i}=\phi_{i}^{a}+\varepsilon_{i} \mu_{i}, \mu_{i} \in\{-1,1\}, i \in I_{\phi}\right\} .
$$

The number of points in $R_{n}$ is $2^{k}$. Let each of these points be indexed by $\phi^{i}, i \in I_{v}$, where

$$
I_{v} \triangleq\left\{1,2, \cdots, 2^{k}\right\} \text {. }
$$




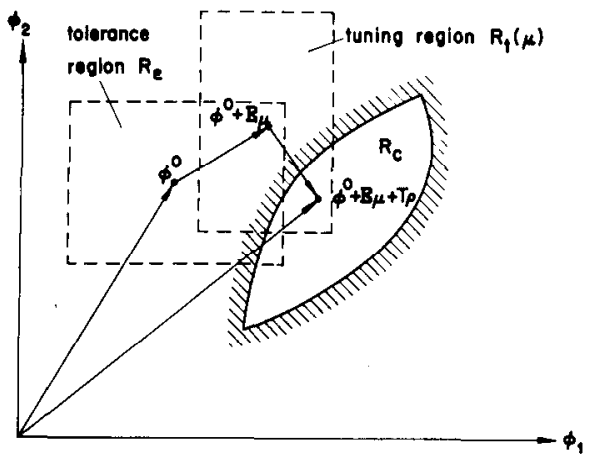

Fig. 1. Illustration of regions $R_{\varepsilon}, R_{t}$, and $R_{c}$. If $\mu=0$ then $R_{t}$ is centered at $\phi^{\circ}$.

Thus

$$
R_{v}=\left\{\phi^{1}, \phi^{2}, \cdots, \phi 2^{k}\right\}
$$

The tuning region $R_{t}(\mu)$ is defined as the set of points

$$
\phi=\phi^{0}+E \mu+T \rho
$$

for all $\rho \in R_{\rho}$, where

$$
T \triangleq\left[\begin{array}{llll}
t_{1} & & & \\
& t_{2} & & \\
& & \ddots & \\
& & & t_{k}
\end{array}\right] .
$$

The components of $\rho$ will be called slack variables since they do not directly contribute to the objective function. Some of the common examples of $R_{\rho}$ are

$$
R_{\rho} \triangleq\left\{\boldsymbol{\rho} \mid-1 \leq \rho_{i} \leq 1, i \in I_{\phi}\right\}
$$

or in the case of one-way tuning or irreversible trimming,

$$
R_{\rho}=\left\{\boldsymbol{\rho} \mid 0 \leq \rho_{i} \leq 1, i \in I_{\phi}\right\}
$$

or

$$
R_{\rho}=\left\{\rho \mid-1 \leq \rho_{i} \leq 0, i \in I_{\phi}\right\} .
$$

Unless otherwise indicated, the case given by (13) is considered.

The constraint region $R_{c}$ is given by

$$
R_{c} \triangleq\left\{\phi \mid g_{i}(\phi) \geq 0, \quad \text { for all } i \in I_{c}\right\}
$$

where

$$
I_{c} \triangleq\left\{1,2, \cdots, m_{c}\right\}
$$

is the index set for the performance specifications and parameter constraints. $R_{c}$ is assumed to be not empty. Other conditions and assumptions will be imposed on $R_{c}$ as the theory is developed further.

The definitions are illustrated in Fig. 1 by a two-dimensional example.

A tunable constraint region is denoted by $R_{c}(\psi)$, where $\psi$ represents other independent variables. Fig. 2 depicts three different regions of an example of $R_{c}(\psi)$. Overlapping of these regions is allowable. The value of $\psi$ may be continuous

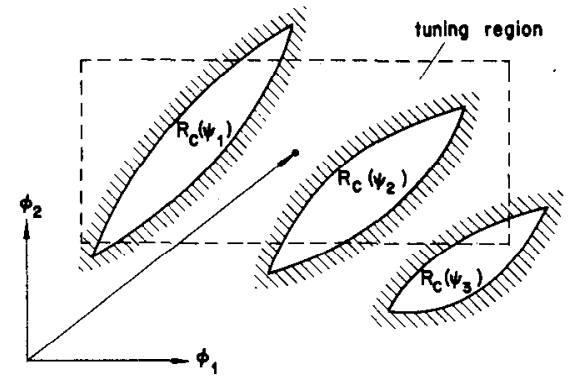

Fig. 2. Example of three different settings of tunable constraint
regions. or discrete. $R_{c}(\psi)=R_{c}$ in the ordinary sense if $\psi$ is a. constant.

\section{The Original Problem $P_{0}$}

The problem may be stated as follows: obtain a set of optimal design values $\left\{\phi^{0}, \varepsilon, t\right\}$ such that any outcome $\left\{\phi^{0}, \varepsilon, \mu\right\}, \mu \in R_{\mu}$, may be tuned into $\boldsymbol{R}_{\mathrm{c}}$ for some $\rho \in \boldsymbol{R}_{\boldsymbol{\rho}}$.

It is formulated as the nonlinear programming problem:

$$
\begin{gathered}
P_{0}: \text { minimize } C\left(\phi^{0}, \varepsilon, t\right) \\
\text { subject to } \phi \in R_{c}
\end{gathered}
$$

where

$$
\phi=\phi^{0}+E \mu+T \rho
$$

and constraints $\phi^{0}, \varepsilon, t \geq 0$, for all $\mu \in R_{\mu}$ and some $\rho \in R_{\rho}$. $C$ is an appropriate function chosen to represent a reasonable approximation to known component cost data.

Stated in an abstract sense, the worst-case solution of the problem must satisfy

$$
R_{t}(\mu) \cap R_{c} \neq \varnothing .
$$

for all $\mu \in R_{\mu}$, where $\varnothing$ denotes a null set.

\section{The Reduced Problem $\boldsymbol{P}_{1}$}

The original problem $P_{0}$ of the preceding section can be reduced by separating the components into effectively tuned and effectively toleranced parameters. Let

$$
\begin{aligned}
I_{\varepsilon} & \triangleq\left\{i \mid \varepsilon_{i}>t_{i}, i \in I_{\phi}\right\} \\
I_{t} & \triangleq\left\{i \mid t_{i} \geq \varepsilon_{i}, i \in I_{\phi}\right\} \\
\varepsilon_{i}^{\prime} & \triangleq \varepsilon_{i}-t_{i}, i \in I_{\varepsilon}
\end{aligned}
$$

and

$$
t_{i}^{\prime} \triangleq t_{i}-\varepsilon_{i}, i \in I_{i}
$$

It is obvious that $I_{t}$ and $I_{\varepsilon}$ are disjoint and $I_{t} \cup I_{\varepsilon}=I_{\phi}$.

Now, consider the problem

$$
\begin{gathered}
P_{1}: \text { minimize } C\left(\phi^{0}, \varepsilon, t\right) \\
\text { subject to } \phi \in R_{c}
\end{gathered}
$$

where

$$
\phi_{i}=\phi_{i}{ }^{0}+ \begin{cases}\varepsilon_{i}^{\prime} \mu_{i}, & \text { for } i \in I_{z} \\ t_{i}^{\prime} \rho_{i}^{\prime}, & \text { for } i \in I_{t}\end{cases}
$$


for all $-1 \leq \mu_{i} \leq 1, i \in I_{\varepsilon}$, and for some $-1 \leq \rho_{i}^{\prime} \leq 1$, $i \in I_{t}$.

\section{Theorem 1}

A feasible solution to the reduced problem $P_{1}$ is a feasible solution to the original problem $P_{0}$.

Proof: Given $\phi^{0}, \varepsilon, t$ we will show that

1) $\varepsilon_{i} \mu_{i}+t_{i} \rho_{i}=\varepsilon_{i}^{\prime} \mu_{i}, i \in I_{\varepsilon}$

2) $\varepsilon_{i} \mu_{i}+t_{i} \rho_{i}=t_{i}^{\prime} \rho_{i}^{\prime}, i \in I_{t}$

under the restrictions on $\mu_{i}, \rho_{i}$, and $\rho_{i}{ }^{\prime}$.

1) Since $\rho_{i}$ can be freely chosen from $-1 \leq \rho_{i} \leq 1$, we can let $\rho_{i}=-\mu_{i}$ giving

$$
\left(\varepsilon_{i}-t_{i}\right) \mu_{i}=\varepsilon_{i}^{\prime} \mu_{i} .
$$

2) For any $-1 \leq \rho_{i}^{\prime} \leq 1$ and all $-1 \leq \mu_{i} \leq 1$, we can choose

$$
-1 \leq \rho_{i}=\frac{\left(t_{i}-\varepsilon_{i}\right) \rho_{i}^{\prime}-\varepsilon_{i} \mu_{i}}{t_{i}} \leq 1, \quad t_{i} \neq 0 .
$$

Thus any point with components represented by (24) of the reduced problem can be represented by (18) of the original problem.

Intuitively, this theorem states the fact that a feasible solution to a restrictive problem is also a feasible solution to an appropriate less restrictive problem. The variable transformation (22) and (23) may be considered as extraneous constraints to be satisfied.

\section{Theorem 2}

A feasible solution to the original problem $P_{0}$ implies a feasible solution to the reduced problem $P_{1}$ if $R_{c}$ is onedimensionally convex [3].

Proof: 1) We note, for $i \in I_{\varepsilon}$, that

$$
\begin{aligned}
\phi_{i}{ }^{0}-\varepsilon_{i}+t_{i} \rho_{i}(-1) & \leq \phi_{i}{ }^{0}-\varepsilon_{i}+t_{i} \leq \phi_{i}{ }^{0}+\left(\varepsilon_{i}-t_{i}\right) \mu_{i} \\
& \leq \phi_{i}{ }^{0}+\varepsilon_{i}-t_{i} \leq \phi_{i}{ }^{0}+\varepsilon_{i}+t_{i} \rho_{i}(1)
\end{aligned}
$$

where $\rho_{i}(-1)$ corresponds to $\mu_{i}=-1$ and $\rho_{i}(1)$ corresponds to $\mu_{i}=1$. If $R_{c}$ is one-dimensionally convex, the following assumption

$\left[\begin{array}{c}\vdots \\ \phi_{i}{ }^{0}-\varepsilon_{i}+t_{i} \rho_{i}(-1) \\ \vdots\end{array}\right], \quad\left[\begin{array}{c}\vdots \\ \phi_{i}{ }^{0}+\varepsilon_{i}+t_{i} \rho_{i}(1) \\ \vdots\end{array}\right] \in R_{c}$

implies that

$$
\left[\begin{array}{c}
\vdots \\
\phi_{i}{ }^{0}+\left(\varepsilon_{i}-t_{i}\right) \mu_{i} \\
\vdots
\end{array}\right] \in R_{c}
$$

where we consider changes in the $i$ th component only and impose the required restrictions on $\mu_{i}$ and $\rho_{i}$.
2) On the other hand, for $i \in I_{t}$, given feasible $\rho_{i}(-1)$ and $\rho_{i}(1)$ such that

$$
\phi_{i}{ }^{0}-\varepsilon_{i}+t_{i} \rho_{i}(-1) \leq \phi_{i}{ }^{0}+\varepsilon_{i}+t_{i} \rho_{i}(1)
$$

there exists a feasible $\rho_{i}{ }^{\prime}$ such that

$$
\begin{aligned}
\phi_{i}{ }^{0}-\varepsilon_{i}+t_{i} \rho_{i}(-1) & \leq \phi_{i}^{0}+\left(t_{i}-\varepsilon_{i}\right) \rho_{i}{ }^{\prime} \\
& \leq \phi_{i}{ }^{0}+\varepsilon_{i}+t_{i} \rho_{i}(1) .
\end{aligned}
$$

This is true for $t_{i}=\varepsilon_{i}$ and can be seen for $t_{i}>\varepsilon_{i}$ by rewriting this inequality as

$$
\frac{-\varepsilon_{i}+t_{i} \rho_{i}(-1)}{t_{i}-\varepsilon_{i}} \leq \rho_{i}^{\prime} \leq \frac{\varepsilon_{i}+t_{i} \rho_{i}(1)}{t_{i}-\varepsilon_{i}} .
$$

Hence, if $R_{c}$ is one-dimensionally convex, the assumption implies that

$$
\left[\begin{array}{c}
\vdots \\
\phi_{i}{ }^{0}+\left(t_{i}-\varepsilon_{i}\right) \rho_{i}{ }^{\prime} \\
\vdots
\end{array}\right] \in R_{c}
$$

Thus, a feasible solution to the original problem can be transformed to a feasible solution of the reduced problem $P_{1}$.

\section{A Geometric Interpretation}

Let us define a projection matrix $\boldsymbol{P}$ as a diagonal matrix such that

$$
\boldsymbol{P} \triangleq\left[\begin{array}{llll}
p_{1} & & & \\
& p_{2} & & \\
& & \ddots & \\
& & & p_{k}
\end{array}\right]
$$

where

$$
p_{i}= \begin{cases}0, & \text { for } i \in I_{t} \\ 1, & \text { for } i \in I_{z}\end{cases}
$$

The projection of a point $\phi$ may be denoted as $\phi_{p}=\boldsymbol{P} \phi$. It may be noted that the projections of two points $\phi^{a}, \phi^{b(j)}=$ $\phi^{a}+\alpha e_{j}$, where $e_{j}$ is the $j$ th unit vector, for $j \in I_{t}$, and some constant $\alpha$, coincide. The projection concept and the introduction of slack variables provide a key to understanding the tuning concept.

Let

$$
R_{\varepsilon t} \triangleq\left\{\phi \mid \phi_{i}^{0}-\varepsilon_{i}^{\prime} \leq \phi_{i} \leq \phi_{i}^{0}+\varepsilon_{i}^{\prime}, i \in I_{\varepsilon}\right\}
$$

and

$$
R_{t \varepsilon} \triangleq\left\{\phi \mid \phi_{i}{ }^{0}-t_{i}{ }^{\prime} \leq \phi_{i} \leq \phi_{i}{ }^{0}+t_{i}{ }^{\prime}, i \in I_{t}\right\}
$$

denote the regions defined by the effectively toleranced and effectively tuned parameters. Then consider the following regions

$$
\begin{aligned}
& R_{\varepsilon t p} \triangleq\left\{\phi_{p} \mid \phi_{p}=P \phi, \phi \in R_{e t}\right\} \\
& R_{c t e} \triangleq R_{c} \cap R_{t e},
\end{aligned}
$$

and

$$
R_{c t \varepsilon p} \triangleq\left\{\phi_{p} \mid \phi_{p}=P \phi, \phi \in R_{c t \varepsilon}\right\}
$$




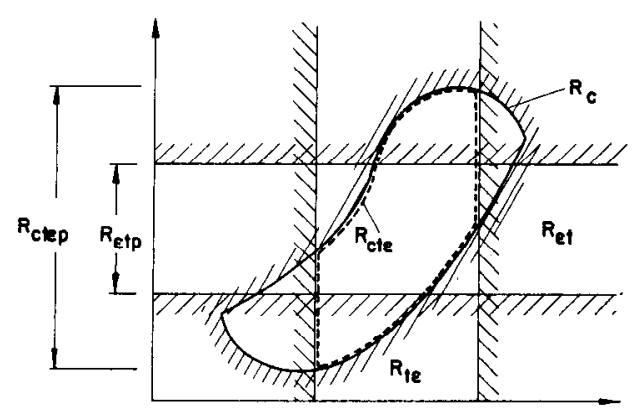

Fig. 3. Geometric interpretation of reduced problem $\boldsymbol{P}_{\mathbf{1}}$.

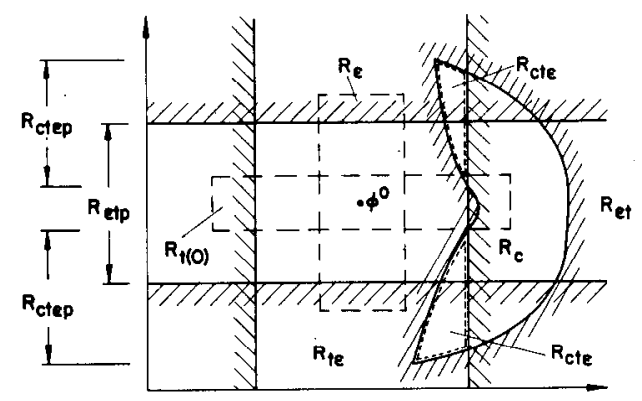

Fig. 4. Example of $R \varepsilon_{t p} \nsubseteq R_{\text {ctep. }} R_{t}(\mu)$ for $\mu=0$ is indicated, for convenience.

Fig. 3 illustrates the definition of the regions. Any point whose components are given by (24) lies in the intersection of $R_{\varepsilon t}$ and $R_{t e}$. Suppose the projection of $R_{c t \varepsilon}$ onto the subspace spanned by the effectively toleranced parameters includes the projection of that point. Then it may be tuned into $R_{c t s}$ by adjusting the value of $\rho_{i}^{\prime}, i \in I_{t}$.

The reduced problem $P_{1}$ may be stated as: solve a pure tolerance problem (i.e., no tuning) in the subspace spanned by the toleranced variables with $R_{\varepsilon t p}$ as the tolerance region and $R_{\text {ctep }}$ as the constraint region. In other words, the regions defined by a feasible solution must satisfy the condition that

$$
R_{e t p} \subseteq R_{c t e p}
$$

Fig. 4 illustrates a case where $R_{\text {etp }} \nsubseteq R_{\text {ctep }}$. An outcome, for example, at $\phi^{0}$ cannot be tuned to $R_{c}$ within the effective tuning range. However, there exists a solution to the original formulation by tuning both componicnts. $R_{c}$ is not onedimensionally convex in this case.

\section{Special Cases}

Case 1: $I_{\varepsilon}=\varnothing$, the Pure Tuning Problem: In this case, $R_{\varepsilon t}$ is the entire space and $\boldsymbol{P}$ is a zero matrix. $R_{\varepsilon t p}$ is a single point at the origin. The problem has a solution if

$$
R_{c t \varepsilon} \neq \varnothing \text {. }
$$

Case 2: $I_{t}=\varnothing$, the Pure Tolerance Problem: In this case, $R_{t \varepsilon}$ is the entire space and $\boldsymbol{P}$ is a unit matrix:

$$
R_{e t p}=R_{e t} \text { and } R_{c t e p}=R_{c t \varepsilon}=R_{c}
$$

The problem has a solution if

$$
R_{\varepsilon t} \subseteq R_{c}
$$

From a tolerance-tuning point of view, the first case is trivial theoretically. Except when there is only one single point $R_{c}$, the pure tuning problem is equivalent to an optimization of the nominal parameter values. On the other hand, the pure tolerance problem is very important from a practical point of view.

\section{Extension of $P_{1}$ for Tunable Constraint Region}

Three types of components can be identified when the constraint region is considered to be tunable. They are a) toleranced components, b) components tuned by the manufacturer, and c) components tunable by the customer. In this case,

$$
\phi \in R_{c}(\psi)
$$

where

$$
\phi_{i}=\phi_{i}^{0}+ \begin{cases}\varepsilon_{i}^{\prime} \mu_{i}, & \text { for } i \in I_{\varepsilon} \\ t_{i}^{\prime} \rho_{i}^{\prime}, & \text { for } i \in I_{t m} \\ t_{i}^{\prime} \rho_{i}^{\prime}(\psi), & \text { for } i \in I_{t c}\end{cases}
$$

where $I_{t m}$ identifies components b) and $I_{t c}$ identifies components $\mathrm{c}$ ).

Setting the $\psi$ to a particular value will control the setting of $\rho_{i}{ }^{\prime}, i \in I_{t c}$, such that $\phi$ will be in that particular constraint region $R_{c}(\psi)$.

\section{The Reduced Problem $P_{2}$}

It is impossible to test all the points in $R_{\text {etp }}$ to be in $\boldsymbol{R}_{\text {etep }}$. In order to make the problem tractable a number of simplifying assumptions could be made to obtain an acceptable solution to the problem with reasonable effort. To this end we replace the continuous range $-1 \leq \mu_{i} \leq 1$ by a discrete set $\mu_{i} \in\{-1,1\}, i \in I_{\varepsilon}$. Now, consider the problem

$$
\begin{gathered}
P_{2}: \text { minimize } C\left(\phi^{0}, \varepsilon, t\right) \\
\text { subject to } \phi \in R_{c}
\end{gathered}
$$

where

$$
\phi_{i}=\phi_{i}{ }^{0}+ \begin{cases}\varepsilon_{i}{ }^{\prime} \mu_{i}, & \text { for } i \in I_{\varepsilon} \\ t_{i}{ }^{\prime} \rho_{i}{ }^{\prime}, & \text { for } i \in I_{t}\end{cases}
$$

for all $\mu_{i} \in\{-1,1\}, i \in I_{\varepsilon}$, and some $-1 \leq \rho_{i}{ }^{\prime} \leq 1, i \in I_{t}$.

Let us define the set of projected vertices (or the vertices of the projected region) by

$$
R_{v p} \triangleq\left\{\phi_{p} \mid \phi_{p}=P \phi, \phi \in R_{v}\right\} .
$$

The condition may be now stated as

$$
R_{v p} \subseteq R_{c t s p}
$$

\section{Theorem 3}

A feasible solution to reduced problem $P_{2}$ implies a feasible solution to reduced problem $P_{1}$ if $R_{c t z p}$ is onedimensionally convex. 
This is a pure tolerance problem in the subspace spanned by the effectively toleranced parameters. For a proof in the tolerance parameter space, see Bandler [3].

\section{The ObJective Functions}

Several objective functions (or cost functions) have been proposed [1]-[5]. In practice, a suitable modeling problem would have to be solved to determine the cost-tolerance relationship. Here, it is assumed that the tolerances and tuning ranges (either absolute or relative) are the main variables and that the total cost of the design is the sum of the cost of the individual components.

The objective function should have the following properties

$$
\begin{array}{ll}
C\left(\phi^{0}, \varepsilon, t\right) \rightarrow c, & \text { as } \varepsilon \rightarrow \infty \\
C\left(\phi^{0}, \varepsilon, t\right) \rightarrow \infty, & \text { for any } \varepsilon_{i} \rightarrow 0 \\
C\left(\phi^{0}, \varepsilon, t\right) \rightarrow C\left(\phi^{0}, \varepsilon\right), & \text { as } t \rightarrow 0 \\
C\left(\phi^{0}, \varepsilon, t\right) \rightarrow \infty, & \text { for any } t_{i} \rightarrow \infty .
\end{array}
$$
form

Suitable objective functions will be, for example, of the

$$
C=\sum_{i=1}^{k} \frac{c_{i}}{x_{i}}+\sum_{i=1}^{k} c_{i}^{\prime} y_{i}
$$

where $x_{i}$ and $y_{i}$ denote the tolerances and tuning ranges, respectively. In the case of relative tolerances or relative tuning ranges $x_{i}=\varepsilon_{i} / \phi_{i}{ }^{0} \times 100, y_{i}=t_{i} / \phi_{i}{ }^{0} \times 100$. We may set the appropriate $c_{i}{ }^{\prime}$ to zero if tuning is considered either free, or fixed or is not required. $c_{i}$ may be set to zero if the corresponding tolerance is fixed.

\section{Mathematical Example}

Consider the constraints

$$
\begin{array}{r}
\phi_{2}-\phi_{1}-2 \geq 0 \\
-\phi_{2}{ }^{2}+16 \phi_{1} \geq 0
\end{array}
$$

A convex region $R_{c}$ is defined by these constraints.

We will take $R_{\mu}$ as an infinite set of discrete points $\mu(i)$, $i=1,2, \cdots$, where $-1 \leq \mu_{1}(i) \leq 1$ and $-1 \leq \mu_{2}(i) \leq 1$. Thus a relevant problem may be formulated as follows. Minimize

$$
C=\frac{1}{\varepsilon_{1}}+\frac{1}{\varepsilon_{2}}
$$

with respect to $\varepsilon_{1}, \varepsilon_{2}, \phi_{1}{ }^{0}$, and $\phi_{2}{ }^{0}$, and subject to

$$
\begin{gathered}
g_{1}=\varepsilon_{1} \geq 0 \\
g_{2}=\varepsilon_{2} \geq 0 \\
g_{3}=\phi_{1}{ }^{0} \geq 0 \\
g_{4}=\phi_{2}{ }^{0} \geq 0 \\
g_{5}(i)=\left(\phi_{2}{ }^{0}+\varepsilon_{2} \mu_{2}(i)\right)-\left(\phi_{1}{ }^{0}+\varepsilon_{1} \mu_{1}(i)\right)-2 \geq 0 \\
i=1,2, \cdots \\
g_{6}(i)=-\left(\phi_{2}{ }^{0}+\varepsilon_{2} \mu_{2}(i)\right)^{2}+16\left(\phi_{1}{ }^{0}+\varepsilon_{1} \mu_{1}(i)\right) \geq 0 \\
i=1,2 ; \cdots
\end{gathered}
$$

where $-1 \leq \mu_{1}(i) \leq 1$ and $-1 \leq \mu_{2}(i) \leq 1$.
Optimality requires that

$$
\begin{gathered}
{\left[\begin{array}{c}
-\frac{1}{\varepsilon_{1}{ }^{2}} \\
-\frac{1}{\varepsilon_{2}{ }^{2}} \\
0 \\
0
\end{array}\right]=\left[\begin{array}{l}
u_{1} \\
u_{2} \\
u_{3} \\
u_{4}
\end{array}\right]+\sum_{i} u_{5}(i)\left[\begin{array}{c}
-\mu_{1}(i) \\
\mu_{2}(i) \\
-1 \\
1
\end{array}\right]} \\
+\sum_{i} u_{6}(i)\left[\begin{array}{c}
-2 \mu_{2}(i)\left(\phi_{2}{ }^{0}+\varepsilon_{2} \mu_{2}(i)\right) \\
16 \\
u_{1} g_{1}=\cdots=u_{4} g_{4}=u_{5}(i) g_{5}(i)=u_{6}(i) g_{6}(i)=0 \\
-2\left(\phi_{2}{ }^{0}+\varepsilon_{2} \mu_{2}(i)\right)
\end{array}\right] \\
u_{1}, \cdots, u_{4}, u_{5}(i), u_{6}(i) \geq 0, \quad i=1,2, \cdots
\end{gathered}
$$

where $u$ denotes a multiplier. To solve the above equations, assume that $\varepsilon_{1}, \varepsilon_{2}, \phi_{1}{ }^{0}$, and $\phi_{2}{ }^{0}$ are not zero, therefore, set $u_{1}, u_{2}, u_{3}$, and $u_{4}$ to zero. Minimize $g_{5}(i)$ of (55) and $g_{6}(i)$ of (56) with respect to $\mu(i)$. This leads, respectively, to

$$
\left(\phi_{2}{ }^{0}-\varepsilon_{2}\right)-\left(\phi_{1}{ }^{0}+\varepsilon_{1}\right)-2 \geq 0
$$

using $\mu(i)=[1-1]^{T}$ and

$$
-\left(\phi_{2}{ }^{0}+\varepsilon_{2}\right)^{2}+16\left(\phi_{1}{ }^{0}-\varepsilon_{1}\right) \geq 0
$$

using $\mu(i)=\left[\begin{array}{ll}-1 & 1\end{array}\right]^{T}$. The optimality conditions (57)-(59) are correspondingly reduced yielding the solution

$$
\begin{aligned}
\varepsilon_{1} & =0.5 \\
\varepsilon_{2} & =0.5 \\
\phi_{1}{ }^{0} & =4.5 \\
\phi_{2}{ }^{0} & =7.5 .
\end{aligned}
$$

Consider next the problem of minimizing

$$
C=\frac{1}{\varepsilon_{2}}
$$

with respect to $t_{1}{ }^{\prime}, \varepsilon_{2}, \phi_{1}{ }^{0}, \phi_{2}{ }^{0}$, and $\rho_{1}(i)$, and subject to

$$
\begin{gathered}
g_{1}=t_{1}{ }^{\prime} \geq 0 \\
g_{2}=\varepsilon_{2} \geq 0 \\
g_{3}=\phi_{1}{ }^{0} \geq 0 \\
g_{4}=\phi_{2}{ }^{0} \geq 0 \\
g_{5}=0.1-\frac{t_{1}{ }^{\prime}}{{\phi_{1}}^{0}} \geq 0 \\
g_{6}(i)=\left(\phi_{2}{ }^{0}+\varepsilon_{2} \mu_{2}(i)\right)-\left({\phi_{1}}^{0}+t_{1}{ }^{\prime} \rho_{1}{ }^{\prime}(i)\right)-2 \geq 0 \\
i=1,2, \cdots \\
g_{7}(i)=-\left(\phi_{2}{ }^{0}+\varepsilon_{2} \mu_{2}(i)\right)^{2}+16\left(\phi_{1}{ }^{0}+t_{1}{ }^{\prime} \rho_{1}{ }^{\prime}(i)\right) \geq 0 \\
g_{8}(i)=1-\rho_{1}{ }^{\prime}(i) \geq 0, \\
g_{9}(i)=1+\rho_{1}{ }^{\prime}(i) \geq 0, \\
i=1,2, \cdots
\end{gathered}
$$


Here, $\varepsilon_{1}$ is considered fixed at 0.5 and there is a maximum effective tuning range of 10 percent. Hence, the first component does not contribute to the cost. The effective tuning range $t_{1}{ }^{\prime}=t_{1}-0.5$ is used as a variable.

The optimality conditions require that

$$
\begin{aligned}
& {\left[\begin{array}{c}
0 \\
-\frac{1}{\varepsilon_{2}{ }^{2}} \\
0 \\
0 \\
0
\end{array}\right]=\left[\begin{array}{l}
u_{1} \\
u_{2} \\
u_{3} \\
u_{4} \\
0
\end{array}\right]+u_{5}\left[\begin{array}{c}
-\frac{1}{\phi_{1}{ }^{0}} \\
0 \\
\frac{t_{1}{ }^{\prime}}{\phi_{10}{ }^{22}} \\
0 \\
0
\end{array}\right]+\sum_{i} u_{6}(i)\left[\begin{array}{c}
-\rho_{1}{ }^{\prime}(i) \\
\mu_{2}(i) \\
-1 \\
1 \\
-t_{1}{ }^{\prime} e_{i}
\end{array}\right]} \\
& +\sum_{i} u_{7}(i)\left[\begin{array}{c}
16 \rho_{1}{ }^{\prime}(i) \\
-2\left(\phi_{2}{ }^{0}+\varepsilon_{2} \mu_{2}(i)\right) \mu_{2}(i) \\
16 \\
-2\left(\phi_{2}{ }^{0}+\varepsilon_{2} \mu_{2}(i)\right) \\
16 t_{1}{ }^{\prime} e_{i}
\end{array}\right] \\
& +\sum_{i} u_{8}(i)\left[\begin{array}{c}
0 \\
0 \\
0 \\
0 \\
-e_{i}
\end{array}\right]+\sum_{i} u_{9}(i)\left[\begin{array}{l}
0 \\
0 \\
0 \\
0 \\
e_{i}
\end{array}\right] \\
& u_{1} g_{1}=\cdots=u_{5} g_{5}=u_{6}(i) g_{6}(i)=\cdots=u_{9}(i) g_{9}(i)=0 \text {, } \\
& i=1,2, \cdots \\
& u_{1}, \cdots, u_{5}, u_{6}(i), \cdots, u_{9}(i) \geq 0, \quad i=1,2, \cdots .
\end{aligned}
$$

Minimize $g_{6}(i)$ of $(65)$ and $g_{7}(i)$ of (66) with respect to $\mu_{2}(i)$. We use $\mu_{2}(i)=-1$ in $(65)$ and $\mu_{2}(i)=1$ in (66) for this purpose. The corresponding $\rho_{1}{ }^{\prime}(i)=-1$ and $\rho_{1}{ }^{\prime}(i)=1$, respectively, are obtained by maximizing $g_{6}(i)$ and $g_{7}(i)$ with respect to $\rho_{1}^{\prime}(i)$. This yields the solution

$$
\begin{aligned}
t_{1}{ }^{\prime} & =0.5432 \\
\varepsilon_{2} & =1.444 \\
\phi_{1}{ }^{0} & =5.4321 \\
\phi_{2}{ }^{0} & =8.3333 .
\end{aligned}
$$

As expected, the inclusion of tunable elements can increase the tolerance on the components. The tolcrance of the second parameter increases from $\varepsilon_{2}=0.5$ to $\varepsilon_{2}=1.444$ when the first component is allowed to have a maximum effective tuning range of 10 percent. This means that an actual absolute tuning of 1.0432 and a tolerance of 0.5 are designed for $\phi_{1}$. The result can only be accomplished by allowing the nominal point to move. For example, the first component moved from 4.5 to 5.4321 , a shift of 20 percent.

\section{FREQUENCY Domain IMPLemENTATION}

Data for a specific problem is contained in a data vector $a^{i}$ which has the form

$$
\boldsymbol{a}^{i} \triangleq\left[\begin{array}{c}
r \\
\mu \\
\psi \\
S \\
w
\end{array}\right], \quad i=1,2, \cdots, m_{a}
$$

where $\psi$ is an independent parameter denoting frequency or any number to identify a particular function for which the vertex $\phi^{r}$ is chosen. $\mu$ is the vector associated with $\phi^{r}$, in particular,

$$
r=1+\sum_{j=1}^{k}\left[\frac{\mu_{j}^{r}+1}{2}\right] 2^{j-1}, \quad \mu_{j}^{r} \in\{-1,1\} .
$$

$m_{a}$ is the total number of distinct vectors $\boldsymbol{a}^{i} . S$ is a specification and $w$ a weighting factor associated with each $\psi$. In our present work,

$$
w= \begin{cases}+1, & \text { if } S \text { is an upper specification } \\ -1, & \text { if } S \text { is a lower specification. }\end{cases}
$$

The performance constraints may now be formulated in the form of

$$
g=w(S-F) \geq 0
$$

with appropriate subscripts. $F$ is the circuit response function evaluated at sample point $\psi$ and point $\phi$ which is given by

$$
\phi=P \phi^{r}+\sum_{j \in I_{s}}\left(\phi_{j}{ }^{0}+t_{j}^{\prime} \rho_{j}{ }^{\prime}(r)\right) e_{j}
$$

The projection matrix $\boldsymbol{P}$ and the index sets $I_{t}$ and $I_{s}$ are fixed for a particular problem. They are determined before optimization takes place.

Let the $n$ optimization variables be denoted by $x$ including the variable nominal values, tolerances, tuning variables and all the appropriate slack variables $\rho_{j}^{\prime}(r)$, $j \in I_{t}, r \in I_{v}$. Let $m$ be the total number of constraints which include the performance specifications, slack variable bounds, parameter bounds, and any other extra constraints not considered above. In general, for linear network design in the frequency domain

$$
n=k_{0}+k_{\varepsilon}+k_{t}\left(1+n_{v}\right)
$$

and

$$
m=\left[\sum_{i=1}^{n_{\psi}} n_{v}(i)\right]+2 k_{t} n_{v}+\cdots
$$

where $k_{0}, k_{\varepsilon}$, and $k_{t}$ are the number of variable nominal parameters, toleranced and tuned parameters, respectively; $n_{v} \leq 2^{k \varepsilon}$ is the number of distinct vertices chosen; $n^{\psi}$ is the number of frequency points considered; $n_{v}(i)$ is the number of vertices chosen at the $i$ th frequency point and $2 k_{t} n_{v}$ is the number of slack variable bounds. 


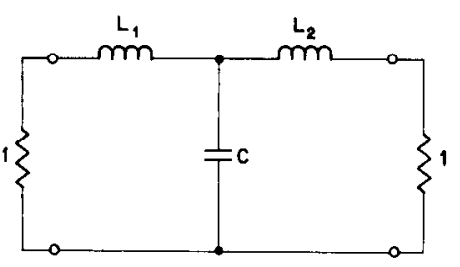

Fig. 5. Circuit for $L C$ low-pass filter example.

TABLE I SPECIFICATIONS FOR $L C$ LOW-PASS FILTER

\begin{tabular}{ccccc}
\hline $\begin{array}{c}\text { Frequency } \\
\text { Range } \\
\text { (rad/s) }\end{array}$ & $\begin{array}{c}\text { Sanple } \\
\text { Points } \\
\text { (rad/s) }\end{array}$ & $\begin{array}{c}\text { Insertion Loss } \\
\text { Specification } \\
\text { (dB) }\end{array}$ & Type & $\begin{array}{c}\text { Weight } \\
\text { wh }\end{array}$ \\
\hline $0-1$ & $0.45,0.50,0.55,1.0$ & 1.5 & upper (passband) & +1 \\
2.5 & 2.5 & 25 & lower (stopband) & -1 \\
\hline
\end{tabular}

TABLE II

Data for Low-Pass Filter

\begin{tabular}{cccccc}
\hline & 1 & 2 & 3 & 4 & 5 \\
\hline$r$ & 6 & 6 & 0 & 8 & 1 \\
& +1 & +1 & +1 & +1 & -1 \\
$\psi$ & -1 & -1 & -1 & +1 & -1 \\
& +1 & +1 & +1 & +1 & -1 \\
$\psi$ & 0.15 & 0.50 & 0.55 & 1.0 & 2.5 \\
$s$ & 1.5 & 1.5 & 1.5 & 1.5 & 25 \\
$w$ & 1 & 1 & 1 & 1 & -1 \\
\hline
\end{tabular}

\section{Low-Pass Filter}

The $L C$ low-pass filter shown in Fig. 5 is considered [5], [6]. Table I summarizes the specifications. The critical vertices used in the data vector $\boldsymbol{a}^{i}$ can be obtained from published vertex selection schemes [6]. These schemes utilize first partial derivative information at some local points or local regions to predict the worst vertices. Very often updating of $\boldsymbol{a}^{i}$ is required at suitable intervals. In this case, the numerical experience we have gained previously from the tolerance problems [5], [6] allows us to chose the minimal set of vertices. These are: $\phi^{6}$ at $\psi=0.45,0.50,0.55 \mathrm{rad} / \mathrm{s}$; $\phi^{8}$ at $\psi=1.0 \mathrm{rad} / \mathrm{s}$ and $\phi^{1}$ at $\psi=2.5 \mathrm{rad} / \mathrm{s}$, where $\phi=$ $\left[L_{1} C L_{2}\right]^{T}$. Updating was not required in this example except when all the three components are toleranced and tuned simultaneously. Table II summarizes the data for the filter.

Several cases have been studied [9] but the results of the case $L_{1}$ tuned with $C$ and $L_{2}$ toleranced will be presented. The objective function used is based on the relative tolerances of $C$ and $L_{2}$ in the form

$$
C=\frac{x_{2}}{x_{5}^{2}}+\frac{x_{3}}{x_{6}^{2}}
$$

where, assuming $t_{C}=t_{L_{2}}=0$, and some fixed value of $\varepsilon_{L_{1}}$, we take

$$
\begin{aligned}
x_{1} & =\phi_{1}{ }^{0}=L_{1}{ }^{0} \\
x_{2} & =\phi_{2}{ }^{0}=C^{0} \\
x_{3} & =\phi_{3}{ }^{0}=L_{2}{ }^{0} \\
x_{4}{ }^{2} & =t_{1}{ }^{0}=t_{L_{1}}-\varepsilon_{L_{1}} \\
x_{5}{ }^{2} & =\varepsilon_{2}=\varepsilon_{C} \\
x_{6}{ }^{2} & =\varepsilon_{3}=\varepsilon_{L_{2}} .
\end{aligned}
$$

The cost of element $L_{1}$ is assumed fixed. It, therefore, is not included in (78). The last three transformations are chosen to avoid changes of sign. There are three distinct projected vertices: $\phi_{p}{ }^{6}, \phi_{p}{ }^{8}$, and $\phi_{p}{ }^{1}$. The projection matrix in this case is

$$
\boldsymbol{P}=\left[\begin{array}{lll}
0 & & \\
& 1 & \\
& & 1
\end{array}\right] \text {. }
$$

Therefore, the other variables may be identified as

$$
\begin{aligned}
& x_{7}=\rho_{1}{ }^{\prime}(6) \\
& x_{8}=\rho_{1}{ }^{\prime}(8) \\
& x_{9}=\rho_{1}{ }^{\prime}(1) .
\end{aligned}
$$

Substituting the numerical values from Table II into (75) we have the following:

$$
\begin{aligned}
a^{1}, a^{2}, a^{3} & \Rightarrow \phi=P \phi^{6}+\left(\phi_{1}{ }^{0}+t_{1}{ }^{\prime} \rho_{1}{ }^{\prime}(6)\right) e_{1} \\
& =\left[\begin{array}{c}
x_{1}+x_{4}{ }^{2} x_{7} \\
x_{2}-x_{5}{ }^{2} \\
x_{3}+x_{6}{ }^{2}
\end{array}\right] \\
\boldsymbol{a}^{4} & \Rightarrow \phi=P \phi^{8}+\left(\phi_{1}{ }^{0}+t_{1}{ }^{\prime} \rho_{1}{ }^{\prime}(8)\right) e_{1} \\
& =\left[\begin{array}{c}
x_{1}+x_{4}{ }^{2} x_{8} \\
x_{2}+x_{5}{ }^{2} \\
x_{3}+x_{6}{ }^{2}
\end{array}\right] \\
\boldsymbol{a}^{5} & \Rightarrow \phi=P \phi^{1}+\left(\phi_{1}{ }^{0}+t_{1}{ }^{\prime} \rho_{1}{ }^{\prime}(1)\right) e_{1} \\
& =\left[\begin{array}{c}
x_{1}+x_{4}{ }^{2} x_{9} \\
x_{2}-x_{5}{ }^{2} \\
x_{3}-x_{6}{ }^{2}
\end{array}\right] .
\end{aligned}
$$

The performance specifications $g_{i}, i=1,2, \cdots, 5$, may now be formed. Additional constraints are given by

$$
\begin{aligned}
g_{5+2 i-1} & =1+x_{6+i} \\
g_{5+2 i} & =1-x_{6+i} \\
g_{12} & =t_{r}-x_{4}^{2} / x_{1} .
\end{aligned} \quad i=1,2,3
$$

The last constraint $g_{12}$ is designed to limit the effective tuning range to $t_{r}$.

The resulting nonlinear programming problem (9 variables, 12 constraints) is solved by a least $p$ th optimization algorithm due Charalambous [10] and the quasiNewton method developed by Fletcher [11] and Gill and Murray [12]. The starting point corresponds to the optimally toleranced nominal point and arbitrary small tolerance and tuning values. Typically, a few hundred function evaluations with less than $30 \mathrm{~s}$ of CDC 6400 computing time is 
TABLE III

$L_{1}$ TUNEd, $C$ AND $L_{2}$ TOleranced

\begin{tabular}{|c|c|c|c|}
\hline Parameters & $t_{r}=0.2$ & ${ }^{t_{r}}=0, \mathrm{I}$ & ${ }^{t}{ }_{r}=0.05$ \\
\hline $\mathrm{L}_{1}^{0}$ & 2.0932 & 2.2442 & 2.1953 \\
\hline$c^{0}$ & 0.9360 & 0.9059 & 0.9062 \\
\hline$L_{2}^{o}$ & 1.7718 & 1.7569 & 1.7920 \\
\hline $100 t_{1}^{\prime} / L_{1}^{0}$ & $20.00 \%$ & $10.00 \%$ & $5.00 \%$ \\
\hline $100 \varepsilon_{2} / \mathrm{C}^{0}$ & $15.99 \%$ & 14.233 & $12.60 \%$ \\
\hline $100 \varepsilon_{3} / L_{2}^{0}$ & $21.62 \%$ & $18.41 \%$ & $16.23 \%$ \\
\hline$o_{1}^{\prime}(6)$ & & -1.0000 & \\
\hline$\dot{0}_{1}^{\prime}(8)$ & & -1.0000 & \\
\hline $0_{1}(1)$ & & 1.0000 & \\
\hline
\end{tabular}

† For the optimally toleranced solution [5] $L_{1}{ }^{0}=L_{2}{ }^{\circ}=1.9990$, $C^{0}=0.9056,100 \varepsilon_{1} / L_{1}{ }^{\circ}=100 \varepsilon_{3} / L_{2}{ }^{0}=9.89 \%, 100 \varepsilon_{2} / C^{0}=7.60 \%$.

required. Table III summarizes the results. Three different tuning ranges are used. The 5-percent tuning of $L_{1}$ increases the tolerances of the other two components by as much as 65 percent. A 10-percent positive and negative shift is obtained for $L_{1}{ }^{0}$ and $L_{2}{ }^{0}$, respectively. $C^{0}$ is shifted slightly. The slack variables assume values of $-1,-1$, and 1 , indicating that the worst cases do occur at the vertices and, correspondingly, the tuning is set to extreme values.

Tuning of $C$ presents a very interesting case. The symmetry property observed in the pure tolerance problem is preserved. Due to this symmetry, a 5-percent tuning range of $C$ produces an increase of 90 percent in the tolerances of $L_{1}$ and $L_{2}$.

Suppose the designer has no prior knowledge of the choice of the tuning component. We consider an objective function' of the form

$$
C=\sum_{i=1}^{3}\left[\frac{\phi_{i}^{0}}{\varepsilon_{i}}+c \frac{t_{i}}{\phi_{i}{ }^{0}}\right]
$$

One additional vertex $\phi^{3}$ is considered in order to bound the solution during optimization. We omit details of the constraints, and summarize the final results in Table IV for different $c$. There are 21 variables and 36 constraints, hence, the computational effort has substantially increased over the previous case. The advantage gained in the general formulation is that the optimization will automatically choose the most appropriate component for tuning, which is $C$ in the objective of (85).

The same designs can be obtained by the reduced formulation using $C$ as a tuned and toleranced component and $L_{1}$ and $L_{2}$ as toleranced components.

\section{High-Pass Filter}

This problem was suggested by Pinel and Roberts [13]. The circuit diagram is shown in Fig. 6 and the basic specifications for the design are listed in Table V. The insertion loss relative to the loss at $990 \mathrm{~Hz}$ is to be constrained as indicated with resistances $R_{5}$ and $R_{7}$ related to $L_{5}{ }^{\circ}$ and
TABLE IV

OPTIMAL TUNING

\begin{tabular}{|c|c|c|c|}
\hline Parameters & $c=10$ & $c=20$ & $c=50$ \\
\hline$L_{1}^{0}=L_{2}^{0}$ & 1.8440 & 1.9221 & 2.0492 \\
\hline$c^{0}$ & 1.1730 & 1,0486 & 0.9069 \\
\hline $100 \varepsilon_{1} / L_{1}^{0}=100 \varepsilon_{3} / L_{2}^{0}$ & $31.62 \%$ & 23.84 i & $16.15 \%$ \\
\hline $100 \varepsilon_{2} / c^{0}$ & $31,62 \%$ & $22,36:$ & $14,14 \%$ \\
\hline $100 \mathrm{t}_{1} / \mathrm{L}_{1}^{0}-100 \mathrm{t}_{3} / \mathrm{L}_{2}^{0}$ & $2.54 \%$ & $0.00 \%$ & $0.00:$ \\
\hline $100 \mathrm{t}_{2} / \mathrm{C}^{0}$ & $54,31 \%$ & $35.89 \%$ & $14.14:$ \\
\hline$o_{1}(6)$ & -1.0000 & -0.7165 & 0.9743 \\
\hline$o_{2}^{(6)}$ & 0.1645 & 0.2466 & 1.0000 \\
\hline$P_{3}(6)$ & -1.0000 & -0.9992 & -0.9846 \\
\hline$\rho_{1}(8)$ & -1.0000 & -1.0000 & -0.8813 \\
\hline$P_{2}(8)$ & -1.0000 & -1.0000 & -1.0000 \\
\hline$\rho_{3}(8)$ & -1.0000 & -1.0000 & -0.9876 \\
\hline$p_{1}(1)$ & 1.0000 & 0.9887 & 0.9933 \\
\hline$p_{2}(1)$ & 1.0000 & 1.0000 & 1.0000 \\
\hline$p_{3}(1)$ & 1.0000 & 0.9989 & 0.9029 \\
\hline$o_{1}(3)$ & 1.0000 & 0.8433 & -0.6051 \\
\hline${ }^{\circ}{ }_{2}(3)$ & -0.1645 & -0.1468 & 0.6434 \\
\hline$p_{3}(3)$ & 1.0000 & 0.8944 & 0.6441 \\
\hline $100 \varepsilon_{1}^{1} / L_{1}^{0}=100 \varepsilon_{3}^{\prime} / L_{2}^{0}$ & $29.08 \%$ & $23.84 \%$ & $14.14 \%$ \\
\hline $100 \mathrm{t}_{2}^{\prime} / \mathrm{c}^{0}$ & 22.69 & $13.53 \%$ & $0.00 \%$ \\
\hline $\mathrm{n}=21$ & \multicolumn{2}{|c|}{$m=36$} & \\
\hline
\end{tabular}

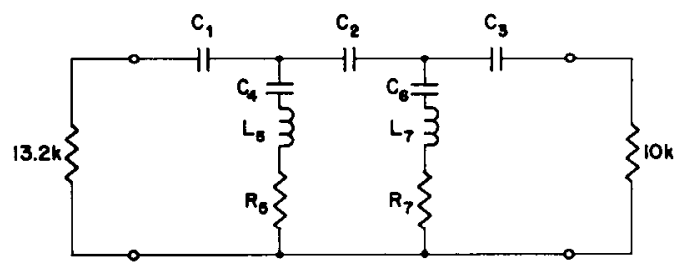

Fig. 6. High-pass filter.

TABLE $V$

Specifications for High-Pass Filter

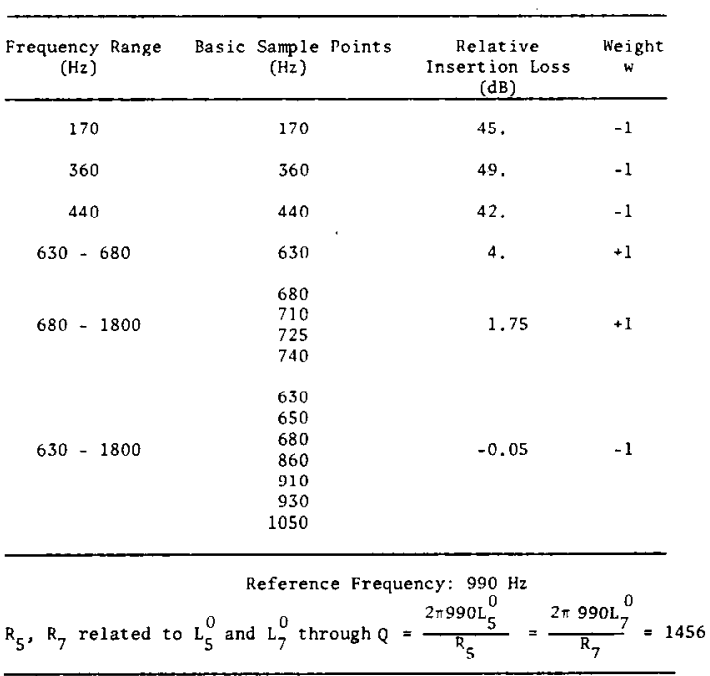


$L_{7}{ }^{\circ}$ with constant $Q$. The terminations are fixed, the designable parameters being $C_{1}, C_{2}, C_{3}, C_{4}, L_{5}, C_{6}$, and $L_{7}$. The objective function throughout was taken as

$$
C=\sum_{i=1}^{7} \frac{\phi_{i}^{0}}{\varepsilon_{i}}
$$

where

$$
\phi^{0}=\left[\begin{array}{l}
C_{1}{ }^{0} \\
C_{2}{ }^{0} \\
C_{3}{ }^{0} \\
C_{4}{ }^{0} \\
L_{5}{ }^{0} \\
C_{6}{ }^{0} \\
L_{7}{ }^{0}
\end{array}\right] \quad \varepsilon=\left[\begin{array}{l}
\varepsilon_{C_{1}} \\
\varepsilon_{C_{2}} \\
\varepsilon_{C_{3}} \\
\varepsilon_{C_{4}} \\
\varepsilon_{L_{3}} \\
\varepsilon_{C_{6}} \\
\varepsilon_{L_{7}}
\end{array}\right]
$$

The optimization package used here is DISOPT [14], which has been previously employed in worst-case tolerance problems [6]. The same quasi-Newton unconstrained minimization procedure as for the work described in the previous section is incorporated into DISOPT. The extrapolation feature [15] was chosen to accelerate convergence to the constrained optimum.

Verification of the designs to be described was carried out using all $2^{7}$ vertices plus the nominal point at $170,360,440$, $630-680$, and $680-1800 \mathrm{~Hz}$. Forty-two logarithmically spaced points were taken for the latter interval, and 8 for the former interval.

Table VI indicates the effort required to obtain the results of Table VII. Because of the complexity of the problems preliminary runs of the program were required before the final number of constraints were established. This information along with a realistic assessment of cost is given.

\section{Case 1: No Tuning $(\boldsymbol{t}=\mathbf{0})$}

Table VI summarizes the particular frequencies, specifications and the particular vertex number employed to obtain the final tolerances listed in Table VII. Table VII also lists the shifts in nominal parameter values with respect to those of an uncentered design [7], [13].

\section{Case 2: 3 Percent Tuning for $L_{5}$}

Results corresponding to the ones for Case 1 are tabulated in Tables VI and VII. Note that all the tolerances have increased. Fig. 7 shows the nominal response as well as the worst upper and lower outcomes based on all $2^{7}$ vertices.

A more detailed verification of the results was made. Sixty logarithmically spaced points were taken from the critical region $630-680 \mathrm{~Hz}$ as well as 40 from $600-630 \mathrm{~Hz}$. All the vertices were checked plus the nominal point, followed by 4000 Monte Carlo simulations uniformly distributed in the effective tolerance region. No violations were detected, and the upper and lower limits of response given by the vertices bounded the results from the Monte Carlo analysis except at $638.2 \mathrm{~Hz}$, where the lowest relative loss obtained from the vertices was $-0.0243 \mathrm{~dB}$, whereas the Monte Carlo analysis yielded $-0.0246 \mathrm{~dB}$.

As a further check on the optimality of these results, $L_{5}$
TABLE VI

\begin{tabular}{|c|c|c|c|c|c|c|}
\hline \multirow{2}{*}{$\begin{array}{l}\text { Frequency } \\
\qquad(\mathrm{Hz})\end{array}$} & \multirow{2}{*}{$\stackrel{S}{S}$} & \multirow{2}{*}{$w$} & \multicolumn{4}{|c|}{ Vertex Number } \\
\hline & & & $\begin{array}{l}\text { Case l } \\
\text { No Tuning }\end{array}$ & $\begin{array}{c}\text { Case } 2 \\
\mathrm{~L}_{5} \text { Tuned }\end{array}$ & $\begin{array}{c}\text { Case } 3 \\
\mathrm{~L}_{5} \text { and } \mathrm{L}_{7} \text { Tuned }\end{array}$ & $\begin{array}{r}\text { Case } 4 \\
L_{7} \text { Tuned }\end{array}$ \\
\hline 170 & 45 & -1 & 8 & 8 & 8 & 8 \\
\hline 360 & 49 & -1 & 48 & 48 & 48 & 48 \\
\hline 440 & 42 & -1 & 128 & 128 & 128 & 128 \\
\hline 630 & 4 & +1 & 1 & 1 & 1 & 1 \\
\hline 630 & -0.05 & -1 & $\begin{array}{l}60,100,104 \\
108,120,126\end{array}$ & $\begin{array}{l}58,60,100 \\
104,108,120 \\
126\end{array}$ & $60,108,120$ & $\begin{array}{l}60,87,95 \\
100,104,108, \\
120,126\end{array}$ \\
\hline 637 & -0.05 & -1 & - & - & - & 87 \\
\hline 640 & -0.05 & -1 & - & 58 & 108 & $52,58,60$ \\
\hline 643 & -0.05 & -1 & - & - & - & $85,93,117$ \\
\hline 650 & -0.05 & -1 & $\begin{array}{l}\text { nomina } 1,12 \\
50,58,102\end{array}$ & $\begin{array}{l}\text { nomina } 1,12 \\
34,42,50,58 \\
102,106,126\end{array}$ & $\begin{array}{l}\text { nominal, } 12,34 \\
42,44,58,106 \\
126\end{array}$ & $\begin{array}{l}\text { nomina } 1,12, \\
36,42,50,58, \\
85,93,94, \\
102,106,126\end{array}$ \\
\hline 658 & -0.05 & -1 & - & - & 42 & $58,69,85$ \\
\hline 665 & -0.05 & -1 & - & - & 34,42 & 34,58 \\
\hline 670 & -0.05 & -1 & - & - & - & 2 \\
\hline 680 & 1.75 & +1 & 123 & 123 & 123 & 123 \\
\hline 680 & -0.05 & -1 & 2,6 & 2,6 & 2.6 & 2,6 \\
\hline 710 & 1.75 & +1 & 43,83 & 43,83 & $43,83,123$ & 43,83 \\
\hline 725 & 1.75 & +1 & 43,83 & 43,83 & 43,83 & 43.83 \\
\hline 730 & 1.75 & +1 & - & - & 43,83 & 43 \\
\hline 740 & 1.75 & +1 & $4.3,83$ & $4.3,8.3$ & $4.3,83$ & 43,83 \\
\hline 860 & -0.05 & -1 & 118,126 & 118,126 & 118,126 & 118,126 \\
\hline 910 & -0.05 & -1 & $118,1.26$ & 118,126 & 118,126 & 118,126 \\
\hline 930 & -0.05 & -1 & 118,126 & 118,126 & 118,126 & 118,126 \\
\hline 1040 & -0.05 & -1 & - & - & - & 3 \\
\hline 1050 & -0.05 & -1 & 3 & 3 & 3 & 3 \\
\hline \multicolumn{2}{|c|}{$\begin{array}{l}\text { Number of Response } \\
\text { Constraints }\end{array}$} & & 31 & 37 & 37 & 55 \\
\hline \multicolumn{2}{|c|}{$\begin{array}{l}\text { Tota1 Number of } \\
\text { Constraints m }\end{array}$} & & 45 & 51 & 51 & 69 \\
\hline Number of & ariables & $n$ & 14 & 14 & 14 & 14 \\
\hline \multicolumn{2}{|c|}{ Starting Point } & & $\begin{array}{l}\text { Given } \\
\text { by Pinel [13] }\end{array}$ & Optimum & for & Casel \\
\hline \multicolumn{2}{|c|}{$\begin{array}{l}\text { Number of Trial } \\
\text { Runs }\end{array}$} & & 3 & 1 & 2 & 1 \\
\hline \multicolumn{2}{|c|}{$\begin{array}{l}\text { Total Computing } \\
\text { Effort (min)t }\end{array}$} & & 15 & 5 & 6 & 7 \\
\hline $\begin{array}{l}\text { Computing } \\
\text { including }\end{array}$ & $\begin{array}{l}\text { ost } \\
\text { rialst }\end{array}$ & & $\$ 94$ & $\$ 31$ & $\$ 37$ & $\$ 44$ \\
\hline
\end{tabular}

Data for Optimization of High-Pass Filter

$\dagger$ On a CDC 6400 .

was allowed to be both toleranced and tuned as distinct from being effectively toleranced from the point of view of optimization. The same vertices, an additional $25-\rho$ variables and 50 additional constraints on the $\rho$ variables were used without any significant improvement in the results. The values of the $\rho$ variables confirmed the assumption that $L_{5}$ should be effectively toleranced for 3 percent tuning.

\section{Case 3: 3 Percent Tuning for $L_{5}$ and $L_{7}$}

As indicated by Table VII a further improvement in all tolerances has been obtained. 


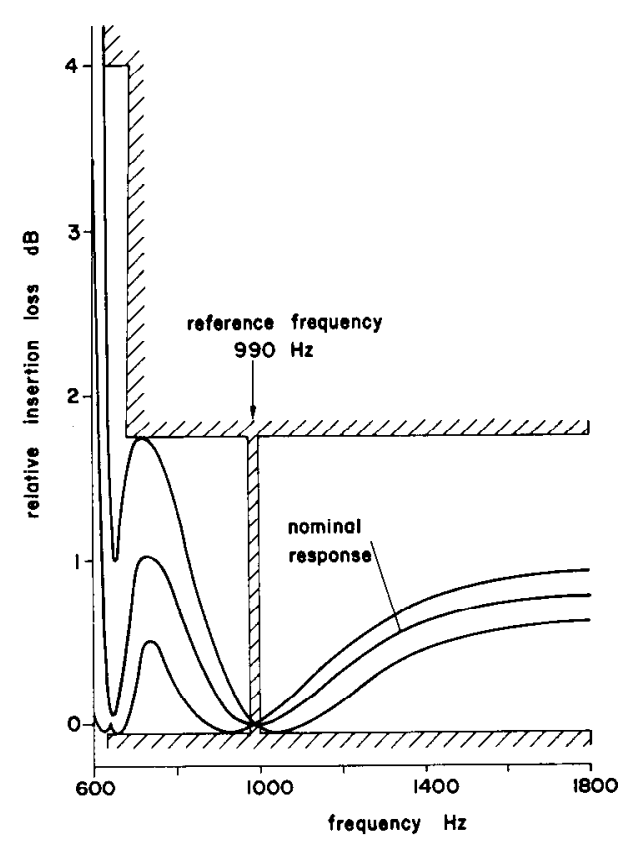

(a)

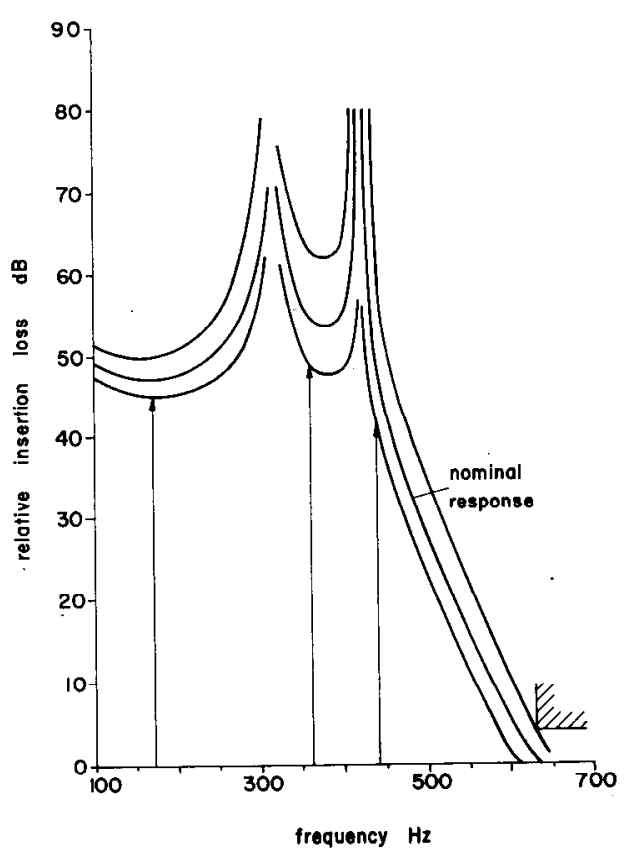

(b)

Fig. 7. (a) Passband details of optimized high-pass filter (Case 2). (b) Stopband details of optimized high-pass filter (Case 2).

\section{Case 4: 3 Percent Tuning for $L_{7}$}

The results for this problem (Table VII) are slightly worse than those for Case 2. A slight violation of the specification at $658 \mathrm{~Hz}$ was detected. We conclude that if only one inductor is to be tuned, $L_{5}$ should be chosen.

\section{Conclusions}

A theory of optimal worst-case design embodying centering, tolerancing and tuning has been presented. The concept of a tunable constraint region that allows variable specifica-
TABLE VII

Results for High-PAss Filter

\begin{tabular}{|c|c|c|c|c|c|}
\hline & Parameters & $\begin{array}{l}\text { Case 1 } \\
\text { No Tuning }\end{array}$ & $\begin{array}{r}\text { Case } 2 \\
\mathrm{~L}_{5} \text { Tuned }\end{array}$ & $\begin{array}{c}\text { Case } 3 \\
\mathrm{~L}_{5} \text { and } \mathrm{L}_{7} \text { Tuned }\end{array}$ & $\begin{array}{l}\text { Case } 4 \\
\mathrm{~L}_{7} \text { Tuned }\end{array}$ \\
\hline$c_{1}$ & $\begin{array}{l}\text { tolerance }(\%) \\
\text { nom. shift }(\%)\end{array}$ & $\begin{aligned} & 5.71 \\
+ & 18.1\end{aligned}$ & $\begin{aligned} & 6.77 \\
+ & 17.8\end{aligned}$ & $\begin{aligned} & 7.90 \\
+ & 18.3\end{aligned}$ & $\begin{aligned} & 6.63 \\
+ & 17.6\end{aligned}$ \\
\hline$c_{2}$ & $\begin{array}{l}\text { tolerance }\left(\frac{\%}{6}\right) \\
\text { nom. shift }\left(\frac{\%}{6}\right)\end{array}$ & $\begin{array}{l}4.3 .3 \\
+16.2\end{array}$ & $\begin{aligned} & 4.97 \\
+ & 15.2\end{aligned}$ & $\begin{aligned} & 5.32 \\
+ & 14.4\end{aligned}$ & $\begin{aligned} & 4.77 \\
+ & 15.3\end{aligned}$ \\
\hline $\mathrm{c}_{3}$ & $\begin{array}{l}\text { tolerance }\left(\frac{\%}{\%}\right) \\
\text { nom. shift }(\%)\end{array}$ & $\begin{aligned} & 4.72 \\
+ & 16.6\end{aligned}$ & $\begin{array}{r}5.81 \\
+18.0\end{array}$ & $\begin{array}{r}7.23 \\
+18.8 \\
\end{array}$ & $\begin{aligned} & 5.83 \\
&+17.8 \\
&\end{aligned}$ \\
\hline $\mathrm{C}_{4}$ & $\begin{array}{l}\text { tolerance }\left(\frac{g}{6}\right) \\
\text { nom. shift }\left(\frac{g}{6}\right)\end{array}$ & $\begin{array}{l}4.54 \\
-3.8\end{array}$ & $\begin{array}{l}5.03 \\
-2.2\end{array}$ & $\begin{array}{l}5.15 \\
-1.2\end{array}$ & $\begin{array}{l}4.78 \\
-3.1\end{array}$ \\
\hline $\mathrm{L}_{5}$ & $\begin{array}{l}\text { tolerance }(\%) \\
\text { nom. shift }(\%)\end{array}$ & $\begin{array}{l}3.29 \\
-3.0\end{array}$ & $\begin{array}{l}3.95 \\
-3.0\end{array}$ & $\begin{array}{l}4.44 \\
-4.3\end{array}$ & $\begin{array}{l}3.82 \\
-4.1\end{array}$ \\
\hline$c_{6}$ & $\begin{array}{l}\text { tolerance }\left(\begin{array}{l}\% \\
6\end{array}\right) \\
\text { nom. shift }\left(\begin{array}{l}\circ \\
y\end{array}\right)\end{array}$ & $\begin{array}{l}6.32 \\
-7.3\end{array}$ & $\begin{array}{l}7.05 \\
5.1\end{array}$ & $\begin{array}{l}7.27 \\
-3.6\end{array}$ & $\begin{array}{l}6.66 \\
-6.0\end{array}$ \\
\hline \multirow[t]{2}{*}{$\mathrm{L}_{7}$} & $\begin{array}{l}\text { tolerance }(\%) \\
\text { nom. shift }(\%)\end{array}$ & $\begin{array}{l}3.64 \\
-6.4\end{array}$ & $\begin{aligned} & 4.34 \\
- & 7.9\end{aligned}$ & $\begin{array}{l}5.04 \\
-7.9\end{array}$ & $\begin{array}{l}4.32 \\
-6.3\end{array}$ \\
\hline & Cost & 157 & 135 & 121 & $138^{*}$ \\
\hline
\end{tabular}

* Violation of specifications. Relative loss $=-0.052 \mathrm{~dB}$ at $658 \mathrm{~Hz}$.

tions as set by the customer has also been incorporated. This may find application, for example, in tunable filters. The purely toleranced and purely tuned problems become special cases. Further simplification has been discussed in the light of one-dimensional convexity.

As expected, the inclusion of tunable elements can increase the tolerances on the components. The results seem to justify the reduction of the general tolerance-tuning problem into one containing effectively toleranced and effectively tuned components, where appropriate. If the separation of the components is not decided in advance, the general problem with the cost function reflecting both tolerances and tuning ranges is appropriate, since an optimization program requires an explicit number of variables and constraints in advance.

A component may be both tuned and toleranced simultaneously. Thus, one can represent the effects of an uncertainty of a tuned component if the tuning range is larger than the tolerance. On the other hand, if the tolerance is larger than the tuning range (see, for example, Table VII), it may be considered to be a toleranced component with some small tuning capacity. The tuning range may or may not appear in the objective function. The different weightings of tuning and tolerancing in the objective exhibit the flexibility of the formulation. With a very heavy weighting in the tuning, we will obtain a solution equivalent to a pure tolerance problem. Zero tuning is automatically indicated by the result of the formulation. Reducing the weighting will increase the tolerance as well as the tuning with a net effect of reducing the effective tolerance $\varepsilon_{i}{ }^{\prime}=\varepsilon_{i}-t_{i}$ until a crossover occurs from effective tolerance to effective tuning. Beyond that, the effective tuning value will continue to increase until a threshold value occurs. Below the threshold, the solution in terms of effective tuning and tolerance problem is unaffected. The tolerances of other components will continue to increase with decreasing weighting on the tuning.

A cost function tending to maximize tolerances and minimizing tuning has been implemented successfully in this context. For the high-pass filter the 3-percent tuning range 
on the inductors was considered free, thus tuning did not enter into the objective function. A reduced problem involving effective tolerances was found adequate since, as shown in Table VII, the colerances exceed the tuning ranges. A good starting point for the tuning problem is a worst-case tolcranced solution. The small tuning ranges in the highpass filter problem meant that relatively small nominal shifts were obtained.

It may be added that, as far as the authors are aware, this seems to be the most general formulation to date dealing with the centering, tolerancing and tuning problems at the design stage.

\section{ACKNOWLEDGMENT}

The authors are indebted to J. F. Pinel and K. A. Roberts of Bell-Northern Research, Ottawa, Canada, for valuable discussions and cooperation, particularly on the high-pass filter problem. The authors also acknowledge discussions with P. Dalsgaard of Aalborg Universitetscenter, Denmark, Dr. E. Della Torre of McMaster University, Hamilton, Ont., Canada, Dr. K. Singhal of the University of Waterloo, Waterloo, Ont., Canada, and the help of J. H. K. Chen, now with Bell-Northern Research.

\section{REFERENCES}

[1] J. F. Pinel and K. A. Roberts, "Tolerance assignment in linear networks using nonlinear programming," IEEE Trans. Circuit Theory, vol. CT-19, Sept. 1972, pp. 475-479.

[2] J. F. Pinel, "Tolerance assignment and network alignment of linear networks in the frequency domain," in IEEE Short Course on Computer Aided Network Design, 73-SC-06, Mar. 1973, pp. $17-25$.

[3] J. W. Bandler, "Optimization of design tolerances using nonlinear programming," J. Optimization Theory and Applications, vol. 14, July 1974 , pp. 99-114.

[4] B. Karafin, "The general component tolerance assignment problem in electrical networks," Ph.D. dissertation, Univ. Pennsylvania, Philadelphia, 1974.

[5] J. W. Bandler and P. C. Liu, "Automated network design with optimal tolerances," IEEE Trans. Circuits Syst., vol. CAS-21, Mar. 1974, pp. 219-222.

[6] J. W. Bandler, P. C. Liu, and J. H. K. Chen, "Worst case network tolerance optimization," IEEE Trans. Microwave Theory Tech., vol. MTT-23, pp. 630-641, Aug. 1975 .

[7] J. F. Pinel, K. A. Roberts, and K. Singhal, "Tolerance assignment in network design,"' in Proc. IEEE Int. Symp. Circuits and Systems, pp. $317-320$.

[8] J. F. Pinel, "Computer-aided network tuning," IEEE Trans. Circuit Theory, vol. CT-18, Jan. 1971, pp. 192-194.

[9] P. C. Liu, "A theory of optimal worst-case design embodying centering, tolerancing and tuning, with circuit applications," Ph.D. dissertation, McMaster Univ., Hamilton, Ont., Canada, 1975.

[10] C. Charalambous, "Nonlinear least $p$ th optimization and nonlinear programming," Dep. Combinatorics and Optimization, Univ. Waterloo, Waterloo, Ont. Canada, Rep. CORR 74-3, Jan. 1974.

[11] R. Fletcher, "FORTRAN subroutines for minimization by quasi-Newton methods," Atomic Energy Research Establishment, Harwell, Berkshire, England, Rep. AERE-R7125, 1972.

[12] P. E. Gill and W. Murray, "Quasi-Newton methods for unconstrained optimization," J. Inst. Math. Appl., vol. 9, 1972, pp. 91-108.

[13] J. F. Pinel, Bell-Northern Research, Ottawa, Ont., Canada, private communication, Aug. 30, 1974.

[14] J. W. Bandler and J. H. K. Chen, "DISOPT-a general program for continuous and discrete nonlinear programming problems," Int. J. Syst. Sci., vol. 6, pp. 665-680. Documented listing available through Faculty of Engineering, McMaster Univ., Hamilton, Ont., Canada, Rep. SOC-29, Mar. 1974.

[15] J. W. Bandler and W. Y. Chu, "Computational merits of extrapolation in least $p$ th approximation and nonlinear programming," Proc. 12th Allerton Conf. Circuit and System Theory, pp. 912-921.

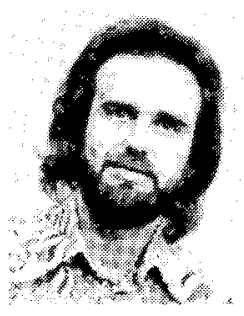

John W. Bandler (S'66-M'66-SM'74) was born in Jerusalem, Palestine, on November 9, 1941. He received the B.Sc. (Eng.) and Ph.D. degrees, both in electrical engineering, from the University of London, Imperial College, London, England, in 1963 and 1967, respectively.

He joined Mullard Research Laboratories, Redhill, Surrey, England, in 1966. From 1967 to 1969 he was a Postdoctorate Fellow and Sessional Lecturer at the University of Manitoba, Winnipeg, Canada. He became Assistant Professor in 1969, Associate Professor in 1971, and Professor in 1974 in the Department of Electrical Engineering at McMaster University, Hamilton, Ont., Canada. He is Coordinator of the Research Group on Simulation, Optimization and Control. He is a contributor to Modern Filter Theory and Design, G. C. Temes and S. K. Mitra, Ed., Wiley-Interscience, 1973. He is author or coauthor of four papers appearing in Computer-Aided Filter Design, G. Szentirmai, Ed., IEEE Press, 1973. He was an Associate Editor of the IEEE Transactions on MicroWave Theory and Technioues (1969-1974). He was Guest Editor of the Special Issue of the IEEE Transactions on Microwave Theory AND TeChNiques on Computer-Oriented Microwave Practices, March 1974.

Dr. Bandler is a member of the Institution of Electrical Engineers (Great Britain) and a member of the Association of Professional Engineers of the Province of Ontario (Canada).

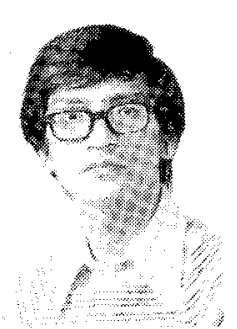

Peter C. Liu (S'74-M'75) was born in Canton, China, on September 22, 1942. He received the B.Sc. and M.Sc. degrees, both in electrical engineering, in 1969 and 1971, respectively, from the University of Manitoba, Winnipeg, Man., Canada. He reccived the Ph.D. degree in electrical engineering from McMaster University, Hamilton, Ont., Canada, in 1975.

$\mathrm{He}$ is now with Bell-Northern Research, Verdun, P.Q., Canada. His work is involved in system modeling and in telecommunication transmission studies. His interests are in circuits systems, numerical techniques, and computer design.

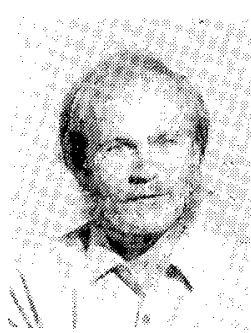

Herman Tromp was born in Antwerp, Belgium, on September 15, 1949. He received the degree in electrical engineering and the degree in communications engineering from the University of Ghent, Ghent, Belgium, in 1972 and 1973, respectively.

In 1972 he became a Research and Teaching Assistant in the Laboratory of Electromagnetism and Acoustics, University of Ghent. He spent the academic year 1974-1975 on leave with the Group on Simulation, Optimization and Control and Department of Electrical Engineering. McMaster University, Hamilton, Canada, as a Rotary Foundation Fellow. He worked in the areas of microwave circuit design and optimization. $\mathrm{He}$ is currently working towards the doctorate in applied sciences at the University of Ghent.

Mr. Tromp is a member of the Royal Society of Flemish Engineers. 